Bull. Korean Math. Soc. 50 (2013), No. 5, pp. 1711-1723

http://dx.doi.org/10.4134/BKMS.2013.50.5.1711

\title{
2-GOOD RINGS AND THEIR EXTENSIONS
}

\author{
YaO WANG AND YANLI REN
}

\begin{abstract}
P. Vámos called a ring $R$ 2-good if every element is the sum of two units. The ring of all $n \times n$ matrices over an elementary divisor ring is 2-good. A (right) self-injective von Neumann regular ring is 2good provided it has no 2-torsion. Some of the earlier results known to us about 2-good rings (although nobody so called at those times) were due to Ehrlich, Henriksen, Fisher, Snider, Rapharl and Badawi. We continue in this paper the study of 2-good rings by several authors. We give some examples of 2-good rings and their related properties. In particular, it is shown that if $R$ is an exchange ring with Artinian primitive factors and 2 is a unit in $R$, then $R$ is 2 -good. We also investigate various kinds of extensions of 2-good rings, including the polynomial extension, Nagata extension and Dorroh extension.
\end{abstract}

\section{Introduction}

Throughout this paper all rings are associative with identity and all modules are unitary. We denote the multiplicative group of units (invertible elements) of the ring $R$ by $U(R)$, the nil radical by $N(R)$ and the Jacobson radical by $J(R)$, and we write $\mathbb{Z}$ for the ring of integers, write $M_{n}(R)$ and $T_{n}(R)$ for the rings of all $n \times n$ matrices and all $n \times n$ upper triangular matrices over the ring $R$, respectively. Recall that a ring $R$ is (von Neumann) regular if for each $a$ in $R$ there exists an $x$ in $R$ such that $a=a x a$. A ring $R$ is called strongly regular [7] if for any $a \in R$ there is an $x \in R$ such that $a^{2} x=a$. A ring $R$ is unit-regular [10] provided that for each $x \in R$ there exists a $u \in U(R)$ such that $x u x=x$. A ring $R$ is $\pi$-regular [8] if for each $a \in R$ there exists an $x \in R$ and a positive integer $n$ such that $a^{n}=a^{n} x a^{n}$. Call a ring $R$ strongly $\pi$-regular $[8]$ if for every element $a \in R$ there exists a positive integer number $n$ (depending on a) and an element $x \in R$ such that $a^{n}=a^{n+1} x$. A ring is elementary division if square matrices can be diagonalized, that is, equivalent to a diagonal matrix (see [11], [18]).

Received December 11, 2012

2010 Mathematics Subject Classification. 16S70, 16 U99.

Key words and phrases. unit, 2-good ring, exchange ring, Artinian primitive factor ring, extensions of rings.

This research is supported by the National Nature Science Foundation of China (11071097, 11101217).

(C)2013 The Korean Mathematical Society 
Vámos [18] called an element $a$ in a ring $R 2-\operatorname{good}$ if $a$ is the sum of two units, and called $R$ 2-good if every element in $R$ is 2-good. In [18], Vámos showed that every ring can be embedded in a 2 -good ring and that a (right) self-injective Von Neumann regular ring is 2-good provided it has no 2-torsion. Some of the earlier results known to us about 2-good rings (although nobody so called at those times) were due to Ehrlich, Henriksen, Fisher, Snider and Raphael. Ehrlich [7, Theorem 7] showed that if $R$ is unit regular and 2 is a unit in $R$, then $R$ is 2-good. Henriksen [11, Theorem 11] showed that the ring of all $n \times n$ matrices over an elementary divisor ring is 2-good. Fisher-Snider [8, Theorem 3] showed that if $R$ is strongly $\pi$-regular and 2 is a unit in $R$, then $R$ is 2-good. Raphael [15, Proposition 2] showed that strongly regula rings are 2-good. 2-good rings were studied under the name $(s, 2)$-ring by Badawi $[1,2]$. [1, Theorem 4] showed that a $\pi$-regular ring $R$ is 2 -good if and only if every idempotent element in $R$ is a sum of two units of $R$, and [2, Theorem 6] showed that if $R$ is abelian $\pi$-regular, then $R$ is 2 -good if and only if $Z / 2 Z$ is not a homomorphic image of $R$. In this paper we continue the study of 2 -good rings, give some examples of 2 -good rings and their related properties, and investigate various kinds of extensions of 2-good rings.

\section{Examples and basic properties}

Example 2.1. Every division ring $R$, which is not isomorphic to $\mathbb{Z}_{2}$, is 2 -good. For $a \in R$, if $a \neq 1$, then $a=1+(a-1)$; if $a=1$, then $1=b+(1-b)$ for $b(\neq 1) \in R$.

Example 2.2. If $R(\neq 0)$ is local and $2 \in U(R)$, then $R$ is 2-good. In fact, if $a \in J(R)$ we have $a=1+(a-1)$, while if $a \notin J(R)$, then $a=\frac{1}{2} a+\frac{1}{2} a$.

Example 2.3. If $X$ is a completely regular Hausdorff space, then the ring $C(X)$ of real valued continuous functions on $X$ is 2-good. Indeed, any $f(x) \in$ $C(X)$ can be written as $f(x)=[f(x) \vee o]+[(f(x) \wedge o)-1]$, a sum of two units in $C(X)$ (see Gillman-Jerison [9, pp.11-15]).

Example 2.4 (Ye [22, Corollary 3.1]). Let $p(\neq 2)$ be a prime number, $\mathbb{Z}_{(p)}=$ $\left\{\frac{m}{n} \mid m, n \in \mathbb{Z},(p, n)=1\right\}$, and $G=(a)=\left\{e, a, a^{2}\right\}$ a cyclic group of order 3 . Then the group ring $\mathbb{Z}_{(p)} G$ is 2 -good.

Recall that a ring $R$ is called clean if every element in $R$ is the sum of an idempotent and a unit in $R$.

Proposition 2.5 (Camillo-Yu [4, Proposition 10]). If $R$ is clean and $2 \in U(R)$, then $R$ is 2-good.

It is worth noting that $2 \in U(R)$ is necessary in Proposition 2.5. Indeed, the ring $R=\mathbb{Z} /(6)$ is clean, and $\overline{2} \notin U(R)$, but it is not 2-good. For, $\overline{5} \in R$ can not be written as a sum of two units.

The concepts of clean rings and 2-good ring are independent of each other. This is illustrated by examples below. 
Example 2.6. Let $R$ be a Boolean ring with more than two elements. For any $x \in R$, we have $x=(x-1)+1$ with $(x-1)^{2}=x-1$ and $1 \in U(R)$. Hence $R$ is a clean ring. Suppose $x \in U(R)$, then $1=x x^{-1}=x^{2} x^{-1}=x$. Thus $U(R)=\{1\}, 1+1=0$, and 0 is the only element which can be written as a sum of units. So $R$ is not 2 -good.

Example 2.7. Let ring $R=\left\{\operatorname{diag}\left(a_{1}, a_{2}, \ldots, a_{n}\right) \mid a_{1}, a_{2}, \ldots, a_{n} \in \mathbb{Z}\right\}$. For any $A=\sum_{i=1}^{n} a_{i} E_{i i} \in R$, if $a_{i_{1}}, a_{i_{2}}, \ldots, a_{i_{k}}=0$ and else where $a_{j} \neq 0$. Without loss of generality we may assume that $a_{1}, \ldots, a_{k}=0$ and $a_{k+1}, \ldots, a_{n} \neq 0$. Put $A_{1}=\sum_{i=1}^{k} 1 E_{i i}+\sum_{j=k+1}^{n} b_{j} E_{j j}, A_{2}=\sum_{i=1}^{k}(-1) E_{i i}+\sum_{j=k+1}^{n} c_{j} E_{j j}$, where $b_{j} \neq 0, c_{j} \neq 0$ and $b_{j}+c_{j}=a_{j}$ for all $k+1 \leq j \leq n$. Then $A=A_{1}+A_{2}$ with $A_{1}, A_{2} \in U(R)$. This prove that $R$ is 2 -good.

But element $B=1 E_{11}+\sum_{i=2}^{n} 0 E_{i i}$ can not be expressed a sum of an idempotent and a unit of $R$. For, if $B=C+D$ with $C^{2}=C=\operatorname{diag}\left(c_{1}, c_{2}, \ldots, c_{n}\right) \in$ $R, D=\operatorname{diag}\left(d_{1}, d_{2}, \ldots, d_{n}\right) \in R$, then from $c_{1}^{2}=c_{1}$ it follows that $c_{1}=1$ and $d_{1}=0$. Thus, $D$ is not a unit of $R$.

Following Goodearl-Menal [10], an associative $\operatorname{ring} R$ is said to satisfy unit 1-stable range if $a R+b R=R$ with $a, b \in R$ implies that there exists some $u \in U(R)$ such that $a+b u \in U(R)$. [10, Theorem 3.1] proved that algebraic algebras over infinite field satisfies unit 1-stable range, and Chen [5, Theorem $2.2]$ showed that if $R$ satisfies unit 1-stable range, then so dose $M_{n}(R)$ for any integer number $n \geq 1$.

Proposition 2.8. Every ring $R$ satisfying unit 1-stable range is 2-good.

Proof. For any $a \in R$, there exists $u \in U(R)$ such that $a+1 \cdot u \in U(R)$ since $a R+1 \cdot R=R$. It follows that $a=(-u)+v$ with $-u, v \in U(R)$.

Corollary 2.9. If $R$ is an algebraic algebra over an infinite field $F$, then $R$ is $2-\operatorname{good}$.

Ehrlich [7] proved that regular rings satisfy the minimum condition on right (left) ideals, semisimple Artinian rings, strongly regular rings and commutative regular rings are all unit regular rings. Fisher-Snider [8, Theorem 1] showed that regular rings with primitive factor Artinian is also unit regular. Thus by [7, Theorem 7] we have the following examples of 2-good rings.

Proposition 2.10. Let $R$ be a ring and $2 \in U(R)$.

(1) If $R$ is semisimple Artinian, then $R$ is 2-good.

(2) If $R$ is strongly regular, then $R$ is 2-good.

(3) If $R$ is commutative regular, then $R$ is 2-good.

(4) If $R$ is a regular ring with primitive factor Artinian, then $R$ is 2-good.

(5) If $R$ is a regular ring satisfies the minimum condition on right (left) ideals, then $R$ is 2-good.

Let $M_{R}$ be a right $R$-module. Following Crawley-Jonsson [6], $M_{R}$ is said to have the exchange property if for every module $A_{R}$ and any two decompositions 
of $A_{R}$

$$
A_{R}=M^{\prime} \bigoplus N=\bigoplus_{i \in I} A_{i}
$$

where $M_{R}^{\prime} \cong M_{R}$, there exist submodules $A_{i}^{\prime} \subseteq A_{i}$ such that

$$
A=M^{\prime} \bigoplus\left(\bigoplus_{i \in I} A_{i}^{\prime}\right) .
$$

Many familiar classes of modules have the exchange property, see ZimmermannHuisgen and Zimmermann [24] for a list of these classes of modules.

Warfield [20] introduced the class of exchange rings. He called a ring $R$ an exchange ring if $R_{R}$ has the exchange property above and proved that this definition is left-right symmetric. The class of exchange rings is quite large. Call a ring $R$ semiregular (semi- $\pi$-regular, semi-strongly $\pi$-regular) if $R / J(R)$ is regular ( $\pi$-regular, strongly $\pi$-regular) and idempotents can be lifted modulo $J(R)$. The following classes of rings are all contained in the class of exchange rings: (1) clean rings (Nicholson [13, Proposition 1.8(1)]); (2) local rings; (3) semiperfect rings; (4) semiregular rings; (5) semistrongly $\pi$-regular rings; (6) semi- $\pi$-regular rings ((2)-(6) see, for example, Stock [16, p. 440] and Tuganbaev $[17$, Theorem 2.11]).

Theorem 2.11. Let $R$ be an exchange ring with Artinian primitive factors. If $2 \in U(R)$, then $R$ is 2-good.

Proof. Assume that $R$ is not a 2-good ring, then there exists $a \in R$ which cannot be expressed as a sum of two units of $R$. Put

$\Omega=\{I \mid I \triangleleft R, \bar{a}$ cannot be expressed as a sum of two units of $R / I\}$,

then $\Omega$ is nonempty. Let $\left\{I_{\alpha}\right\}$ be a chain in $\Omega$ and set $I=\cup_{\alpha} I_{\alpha}$. Then $I \triangleleft R$. If $I \notin \Omega$, then $\bar{a}$ is a sum of two units of $R / I$. Then there exist $u+I, v+I \in U(R / I)$, such that $a+I=(u+I)+(v+I)$. Hence, $\{a-(u+$ $\left.v), u u^{\prime}-1, u^{\prime} u-1, v v^{\prime}-1, v^{\prime} v-1\right\} \subseteq I$ for some $u^{\prime}, v^{\prime} \in R$. Thus there exits $\beta$ such that $\left\{a-(u+v), u u^{\prime}-1, u^{\prime} u-1, v v^{\prime}-1, v^{\prime} v-1\right\} \subseteq I_{\beta}$. So $\bar{a}$ is a sum of two units of $R / I_{\beta}$. This contradicts the choice of $I_{\beta}$, so $I \in \Omega$. By Zorn's Lemma, $\Omega$ contain a maximal element $A$. Let $S=R / A$. The maximality of $A \in \Omega$ implies that $S$ is indecomposable as a ring.

If $J(S) \neq 0$, then $J(S)=B / A$ with $B \supset A$. By the maximality of $A$, $a=a+A \in S$ is the sum of two units of $R / A$. From $S / J(S) \cong R / B$, we have

$$
(a+A)+J(S)=\left[\left(u_{1}+A\right)+J(S)\right]+\left[\left(v_{1}+A\right)+J(S)\right]
$$

with $\left(u_{1}+A\right)+J(S),\left(v_{1}+A\right)+J(S) \in U(S / J(S))$. Since units lift modulo the Jacobson radical of $S$, so that $\overline{u_{1}}=u_{1}+A, \overline{v_{1}}=v_{1}+A \in U(S)$. Thus

$$
\bar{a}=\left(\overline{u_{1}}+\overline{v_{1}}\right)+\bar{r}
$$


for some $\bar{r}=r+A \in J(S)$. Note that $\overline{v_{1}}+\bar{r}=\bar{v}_{1}\left(1+\bar{v}_{1}^{-1} \bar{r}\right) \in U(S)$, hence $\bar{a}=\overline{u_{1}}+\overline{v_{1}}\left(1+{\overline{v_{1}}}^{-1} \bar{r}\right)$ is a sum of two units of $S$. In this final case we have contradicted the choice of $A$. Thus, we see that $J(S)=0$.

Since $R$ is an exchange ring with Artinal primitive factors, by virtue of $\mathrm{Yu}$ [23, Lemma 3.7], $S$ is simple Artinian. By Proposition 2.10(1), $\bar{a}$ can be expressed as a sum of two units of $S$, and it yields a contradiction. Therefor $R$ is 2 -good.

Corollary 2.12. Let $R$ be a regular (resp. $\pi$-regular, semiregular, clean, local, semiperfect, semistrongly $\pi$-regular, semi- $\pi$-regular) ring with primitive factor rings Artinian. If $2 \in U(R)$, then $R$ is 2-good.

Proposition 2.13. Let $F$ be a field and $2 \neq 0, G$ a finite group and char $(F)$ do not divide $|G|$. Then group ring $F G$ is 2-good.

Proof. In virtue of Kelarev [12, Theorem 3.1] (Maschke's Theorem) FG is an semisimple Artinian ring. The result follows by Proposition 2.10.

Recall that a semigroup $S$ is called t.u.p. (two-unique-product) semigroup if, for any nonempty finite subsets $X, Y$ with $|X|+|Y|>2$, there exist at least two elements in $S$ that have unique presentations as $x y$, for some $x \in X, y \in Y$ (see [14]).

Proposition 2.14. Let $K$ be a finite field and $2 \neq 0, S$ be a finite t.u.p. semigroup. Then semigroup ring $K G$ is 2 -good.

Proof. Let $a \in K G$, say $a=k_{1} s_{1}+\cdots+k_{n} s_{n}$ where $k_{i} \in K$ and $s_{i} \in S$ for each $i$. Thus $a \in K_{0} S_{0}$ where $K_{0}$ is the subfield of $K$ generated by $\left\{k_{1}, \ldots, k_{n}\right\}$, and $S_{0}$ is the sub-semigroup of $S$ generated by $\left\{s_{1}, \ldots, s_{n}\right\}$. By hypothesis, $R_{0} S_{0}$ is a finite ring. Hence $R_{0} S_{0}$ is Artinian. According to Okninski [14, Corollary 10.5], $J(K S)=0$ and hence $K S$ is semisimple Artinian ring. The result follows by Proposition 2.10 .

Let $S(R)$ be the nonempty set of all the proper ideals of $R$ generated by central idempotents. Recall that the factor $\operatorname{ring} R / P$ is called a Pierce stalk (see Tuganbaev [17]) of $R$ if $P$ is a maximal element in $S(R)$.

Proposition 2.15. For a ring $R$, the following are equivalent:

(1) $R$ is 2-good.

(2) All homomorphism images of $R$ are 2-good.

(3) All indecomposable factor rings of $R$ are 2 -good.

(4) $R / I$ is 2-good for some ideal $I$ of $R$ contained in $J(R)$.

(5) $A / I$ is 2-good for every proper ideal $I$ of $R$ generated by central idempotents of $R$.

(6) All Pierce stalks of $R$ are 2-good.

Proof. $(1) \Rightarrow(2) \Rightarrow(3),(1) \Rightarrow(2) \Rightarrow(4)$ and $(2) \Rightarrow(5) \Rightarrow(6)$ are trivial. (4) $\Rightarrow(1)$ is a corollary of $[18$, Lemma $2(\mathrm{a})]$. 
$(6) \Rightarrow(1)$ If $R$ is not 2-good. Put

$\Omega=\{I \triangleleft R \mid I$ is a proper ideal generated by central idempotents of $R$ such that $R / I$ is not 2 -good $\}$.

Then $\Omega \neq \phi$ since $\{0\} \in \Omega$. It is easily verified that the union of every chain of ideals from $\Omega$ is contained in $\Omega$. By Zorn's Lemma, $\Omega$ contains a maximal element $J$. We next prove that $J$ is generated by central idempotents. Assume the contrary, then there is a central idempotent $e$ such that $J+e R$ and $J+(1-$ $e) R$ are proper ideals of $R$ and properly contain $J$. Since $(J+e R) \cap(J+(1-$ $e) R)=J,(J+e R)+(J+(1-e) R)=R$ and $R^{2}+(J+e R)=R=R^{2}+(J+(1-$ $e) R$ ), by Chinese Remainder Theorem, $R / J \cong R /(J+e R) \times R /(J+(1-e) R)$. The maximality of $J \in \Omega$ implies that $J+e R$ and $J+(1-e) R$ are not in $\Omega$, hence $R /(J+e R)$ and $R /(J+(1-e) R)$ are 2 -good. It follows that $R / J$ is 2 -good, and it yields a contradiction. Thus we see that $R / J$ is a Pierce stalk. By hypothesis, $R / J$ is 2-good, a contradiction. Therefore $R$ is 2 -good.

$(3) \Rightarrow(1)$ It is similar to $(6) \Rightarrow(1)$, we omit the proof.

Corollary 2.16. Let $e^{2}=e \in R$. Then $e R$ is 2-good if and only if so is eRe.

Proof. Put $\sigma: e R \rightarrow e R e, \sigma(x)=x e$. It is easy to see that $\sigma$ is a epimorphism of rings, $\operatorname{ker} \sigma=e R(1-e), e R / \operatorname{ker} \sigma \cong e R e$, and $\operatorname{ker} \sigma \subseteq J(e R)$ since $(\operatorname{ker} \sigma)^{2}=0$. If $e R e$ is 2-good, by Proposition 2.15(4), $e R$ is 2-good. Conversely, if $e R$ is 2 -good, then $e R e$ is 2-good since it is $\sigma$-homomorphic image of $e R$.

Proposition 2.17. The class of all 2-good rings is an Amitsur-Kurosh radical class.

Proof. Let $P=\{R \mid R$ is a 2 -good ring $\}$. By Proposition 2.15, $P$ is homomorphism closed. If $I$ is an ideal of $R \in P$, and if $I, R / I$ are in $P$, then $1 \in I$, hence $R=I$ is 2 -good. It is easy to see that a union of a chain of $P$-ideals related to a ring $R \in P$ is again a $P$-ideal of $R$.

Finally, we recall that Henriksen [10] called a ring $R(S, n)$-ring if every element of $R$ is a sum of no more than $n$ units. 2-good rings are $(S, 2)$-ring, But the converse is not true. Indeed, $R=\mathbb{Z} /(4)=\{\overline{0}, \overline{1}, \overline{2}, \overline{3}\}$ is a $(S, 2)$-ring but not 2-good.

\section{The extensions of 2-good rings}

Proposition 3.1. (1) $A$ direct product $R=\prod R_{\alpha}$ of 2-good rings $\left\{R_{\alpha}\right\}$ is 2 -good if and only if so is each $R_{\alpha}$.

(2) A finite direct sum $R=\bigoplus_{i=1}^{n} R_{i}$ of 2-good rings $\left\{R_{i}\right\}$ is 2-good if and only if so is each $R_{i}$.

(3) The direct limit $\lim _{\longrightarrow} R_{i}$ is 2-good if and only if so is each $R_{i}, i \in I$.

Proof. These assertions are directly verified. 
If $R$ is a ring and $\alpha: R \rightarrow R$ is a ring endomorphism. Let $R[[x, \alpha]]$ denote the ring of skew formal power series over $R$, that is all formal power series in $x$ with coefficients from $R$ with multiplication defined by $x r=\alpha(r) x$ for all $r \in R$. In particular, $R[[x]]=R\left[\left[x, 1_{R}\right]\right]$ is the ring of formal power series over $R$.

Proposition 3.2. Let $R$ be a ring. Then the ring $R[[x, \alpha]]$ is 2 -good if and only if $R$ is 2-good. In particular, $R[[x]]$ is 2-good if and only if $R$ is 2-good.

Proof. By Proposition 2.15, $R[[x, \alpha]]$ is 2-good, and this gives that

$$
R=R[[x, \alpha]] /(x)
$$

is 2 -good.

Conversely, suppose that $R$ is 2-good. Let $f(x)=\sum_{i=0}^{\infty} a_{i} x^{i} \in R[[x, a]]$. Write $a_{0}=u+v \in U(R)$. Then $f(x)=\left(u+a_{1} x+a_{2} x^{2}+\cdots\right)+v$, where $u+a_{1} x+a_{2} x^{2}+\cdots, v \in U(R[[x, \alpha]])$. Thus $R[[x, \alpha]]$ is 2 -good.

Recall that a ring $R$ is called semicommutative if for all $a, b \in R, a b=0$ implies $a R b=0$. Commutative rings, symmetric rings, reversible rings and one-sided duo rings are all semicommutative (see [3]).

Proposition 3.3. If $R$ is semicommutative, then the polynomial ring $R[x]$ is not 2-good.

Proof. Note that by Xiao-Tong [21, Lemma 3.5],

$U(R[x])=\left\{a_{0}+a_{1} x+\cdots+a_{n} x^{n} \mid a_{0} \in U(R), a_{1}, \ldots, a_{n} \in N(R), n \in N\right\}$.

If $x$ is 2-good, then $x=u(x)+v(x)$, where $u(x)=u_{0}+u_{1} x+\cdots+$ $u_{n} x^{n}, v(x)=v_{0}+v_{1} x+\cdots+v_{m} x^{m} \in U(R[x])$, and $u_{1}, \ldots, u_{n}, v_{1}, \ldots, v_{m} \in$ $N(R), n, m \in N$. It follows that $1=u_{1}+v_{1} \in N(R) \subseteq J(R)$, a contradiction. Thus $R[x]$ is not 2 -good.

Corollary 3.4. If $R$ is commutative (symmetric, reversible, one-side duo), then the polynomial ring $R[x]$ is not 2-good.

Remark 3.5. (1) If $R[x]$ is 2 -good, then so is $R$.

(2) The subring of 2-good ring need not inherit the property.

(3) The polynomial ring $R[x]$ over a 2 -good $\operatorname{ring} R$ need not be 2 -good.

Indeed, if $Q$ is the rational number field, then $Q$ and $Q[[x]]$ are both 2-good by Proposition 3.2, but the polynomial ring $Q[x]$ over $Q$, a subring of $Q[[x]]$, is not 2-good by Corollary 3.4 .

Proposition 3.6. (1) Let $e^{2}=e \in R$. If eRe and $(1-e) R(1-e)$ are both 2-good, then $R$ is 2-good.

(2) Let $e^{2}=e$ is a central idempotent of $R$. Then $R$ is 2-good if and only if so are $e R$ and $(1-e) R$. 
Proof. (1) For convenience write $\bar{e}=1-e$. We use the Pierce decomposition of the ring $R$ :

$$
R=\left(\begin{array}{cc}
e R e & e R \bar{e} \\
\bar{e} R e & \bar{e} R \bar{e}
\end{array}\right)
$$

Let $A=\left(\begin{array}{ll}a & x \\ y & b\end{array}\right) \in R$ with $a \in e R e, b \in \bar{e} R e$. By hypothesis, there exist $u_{1}, u_{2} \in U(e R e)$ with inverse $u_{1}^{-1}$ and $u_{2}^{-1}$ such that $a=u_{1}+u_{2}$. Thus $b-y u_{2}^{-1} x \in \bar{e} R \bar{e}$, so we can write $b-y u_{2}^{-1} x=v_{1}+v_{2}$ where $v_{1}, v_{2} \in U(\bar{e} R \bar{e})$ with inverse $v_{1}^{-1}$ and $v_{2}^{-1}$. Hence

$$
A=\left(\begin{array}{cc}
u_{1}+u_{2} & x \\
y & v_{1}+v_{2}+y u_{2}^{-1} x
\end{array}\right)=\left(\begin{array}{cc}
u_{1} & 0 \\
0 & v_{1}
\end{array}\right)+\left(\begin{array}{cc}
u_{2} & x \\
y & v_{2}+y u_{2}^{-1} x
\end{array}\right)
$$

and it is sufficient to show that $\left(\begin{array}{cc}u_{2} & x \\ y & v_{2}+y u_{2}^{-1} x\end{array}\right)$ is a unit in $R$. To this end compute

$$
\begin{aligned}
& \left(\begin{array}{cc}
e & 0 \\
-y u_{2}^{-1} & \bar{e}
\end{array}\right)\left(\begin{array}{cc}
u_{2} & x \\
y & v_{2}+y u_{2}^{-1} x
\end{array}\right)\left(\begin{array}{cc}
e & -u_{2}^{-1} x \\
0 & \bar{e}
\end{array}\right) \\
= & \left(\begin{array}{cc}
u_{2} & x \\
0 & v_{2}
\end{array}\right)\left(\begin{array}{cc}
e & -u_{2}^{-1} x \\
0 & \bar{e}
\end{array}\right)=\left(\begin{array}{cc}
u_{2} & 0 \\
0 & v_{2}
\end{array}\right) .
\end{aligned}
$$

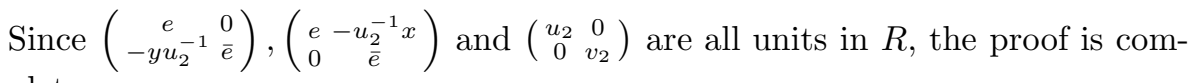
plete.

(2) Suppose $R$ is 2-good. For any er $\in e R$, we have $r=u+v$, where $u, v \in U(R)$ with inverse $u^{-1}$ and $v^{-1}$. It follows that eueu $u^{-1}=e=e v e v^{-1}$. Thus $e u, e v \in U(e R), e r=e u+e v$. Hence $e R$ is 2-good. Note that $e^{\prime}=1-e$ is also a central idempotent of $R$. We know that $(1-e) R$ is 2 -good. The converse is clear by (1).

Vaserstein [19, Theorem 2.8] showed that if $R$ satisfies unit 1-stable rang, then so does $e R e$ for any idempotent $e \in R$. This combined with Proposition 2.8 gives:

Proposition 3.7. If $R$ satisfies unit 1-stable rang, and e is any idempotent in $R$, then eRe is 2-good.

Corollary 3.8. If $1=e_{1}+e_{2}+\cdots+e_{m}$ in a ring $R$ where the $e_{i}$ are orthogonal idempotents and each $e_{i} R e_{i}$ is 2-good, then so is $R$.

Proof. By Proposition 3.6(1) and induction.

Corollary 3.9. If $R$ is 2-good, then so is the matrix ring $M_{n}(R)$ for any positive integer $n$.

Corollary 3.10. If $M=M_{1} \bigoplus M_{2} \bigoplus \cdots \bigoplus M_{n}$ are modules and $\operatorname{End}\left(M_{i}\right)$ is 2 -good for each $i$, then $\operatorname{End}(M)$ is 2-good.

By Proposition 3.6(1) and Proposition 2.15, we obtain: 
Corollary 3.11. If $A$ and $B$ are rings and $M={ }_{B} M_{A}$ is a bimodule, the formal triangular matrix ring $T=\left(\begin{array}{cc}A & 0 \\ M & B\end{array}\right)$ is 2-good if and only if both $A$ and $B$ are $2-\operatorname{good}$.

Recall that a Morita Context denote by $(A, B, M, N, \Psi, \Phi)$ consists of two rings $A, B$, two bimodules ${ }_{A} N_{B},{ }_{B} M_{A}$ and a pair of bimodule homomorphisms $\Psi: N \bigotimes_{B} M \rightarrow A$ and $\Phi: M \bigotimes_{A} N \rightarrow B$ which satisfy the following associativity: $\Psi(v, w) v^{\prime}=v \Phi\left(w, v^{\prime}\right)$ and $\Phi(w, v) w^{\prime}=w \Psi\left(v, w^{\prime}\right)$. These conditions will insure that the set $C$ of generalized matrices

$$
\left(\begin{array}{cc}
a & n \\
m & b
\end{array}\right), a \in A, b \in B, m \in M, n \in N .
$$

will form a ring $C=\left(\begin{array}{cc}A & N \\ M & B\end{array}\right)$, called Morita ring.

Corollary 3.12. If both $A$ and $B$ are 2-good, then so is $C$.

Proof. Take $e=\left(\begin{array}{ll}1 & 0 \\ 0 & 0\end{array}\right)$ and $\bar{e}=1-e=\left(\begin{array}{ll}0 & 0 \\ 0 & 1\end{array}\right)$, we have that $A \cong e C e$ and $B \cong \bar{e} C \bar{e}$. The result follows by Proposition 3.6.

Remark 3.13. The converses of Proposition 3.6(1), Corollary 3.9 and Corollary 3.12 are all not true. For example, by Vámos [18, Proposition 6$], R=M_{2}(\mathbb{Z})$ is 2 -good. Taking $e=\left(\begin{array}{ll}1 & 0 \\ 0 & 0\end{array}\right)$, we have $e R e \cong \mathbb{Z}$, which is not 2 -good. It also shows that the property of 2 -good is not a Morita invariant.

Proposition 3.14. Let $e_{1}, \ldots, e_{n}$ be idempotents of a ring $R$. If $e_{1} R e_{1}, \ldots$, $e_{n} R e_{n}$ are all 2 -good, then so is the ring $\left(\begin{array}{ccc}e_{1} R e_{1} & \cdots & e_{1} R e_{n} \\ e_{n} R e_{1} & \cdots & e_{n} R e_{n}\end{array}\right)$.

Proof. By Proposition 3.6(1), the result holds for $n=2$. Assume inductively that the result holds for $n=k \geq 2$. Let $n=k+1$, and let

$$
\begin{gathered}
B=\left(\begin{array}{ccc}
e_{2} R e_{2} & \cdots & e_{2} R e_{k+1} \\
\cdots & \cdots & \cdots \\
e_{k+1} R e_{2} & \cdots & e_{k+1} R e_{k+1}
\end{array}\right)_{k \times k}, \\
M=\left(\begin{array}{c}
e_{2} R e_{1} \\
\vdots \\
e_{k+1} R e_{1}
\end{array}\right)_{k \times 1}, N=\left(\begin{array}{ccc}
e_{1} R e_{2} & \cdots & e_{1} R e_{k+1}
\end{array}\right)_{1 \times k} .
\end{gathered}
$$

Then $B$ is 2-good. Given $\left(\begin{array}{cc}a & n \\ m & b\end{array}\right) \in\left(\begin{array}{ccc}e_{1} R e_{1} & N \\ M & B\end{array}\right)$, similar to the proof of Proposition $3.6(1)$, we can show that it is a sum of two units.

Corollary 3.15. Let $R$ be a ring. Then the following are equivalent:

(1) $R$ is 2-good.

(2) $R$ has a complete orthogonal set $\left\{e_{1}, \ldots, e_{n}\right\}$ of idempotents such that all $e_{i} R e_{i}$ are 2 -good. 
Proof. $(1) \Rightarrow(2)$ is obvious.

$(2) \Rightarrow(1)$ Construct a map

$$
\Theta: R \rightarrow\left(\begin{array}{ccc}
e_{1} R e_{1} & \cdots & e_{1} R e_{n} \\
\cdots & \cdots & \cdots \\
e_{n} R e_{1} & \cdots & e_{n} R e_{n}
\end{array}\right)
$$

given by $\Theta(r)=\left(\begin{array}{ccc}e_{1} r e_{1} & \ldots & e_{1} r e_{n} \\ \cdots & \ldots & \cdots \\ e_{n} r e_{1} & \cdots & e_{n} r e_{n}\end{array}\right)$. Since $\left\{e_{1}, \ldots, e_{n}\right\}$ is a complete set of orthogonal idempotents, $\Theta$ is a ring isomorphism, we get the result by Proposition 3.14 .

Let $R$ be a ring. Put $Q M_{2}(R)=\left\{\left(\begin{array}{ll}a & b \\ c & d\end{array}\right) \mid a+b=c+d, a, b, c, d \in R\right\}$. Then $Q M_{2}(R)$ is a subring of $M_{2}(R)$.

Theorem 3.16. Let $R$ is 2-good. Then the following statements hold:

(1) For any $n \in N$, the ring $T_{n}(R)$ of $n \times n$ upper triangular matrices over $R$ is 2-good.

(2) $Q M_{2}(R)$ is 2-good.

(3) For any $n \in N, S_{n}(R)=\left\{\left(\begin{array}{ccccc}a_{0} & a_{1} & a_{2} & \cdots & a_{n-1} \\ 0 & a_{0} & a_{1} & \cdots & a_{n-2} \\ 0 & 0 & a_{0} & \cdots & a_{n-3} \\ \ldots & 0 & \cdots & \ldots & \ldots \\ 0 & 0 & 0 & \ldots & \ldots \\ a_{0}\end{array}\right) \mid a_{i} \in R, i=0,1, \ldots, n-1\right\}$ is 2-good.

(4) For any $n \in N, R[x] /\left(x^{n}\right)$ is 2-good, where $\left(x^{n}\right)$ is the ideal generated by $x^{n}$.

Proof. (1) Let $A=\left(a_{i j}\right) \in T_{n}(R)$, where $a_{i j}=0$ if $i>j$. By hypothesis there exist $u_{i}, v_{i} \in U(R)$ such that $a_{i i}=u_{i}+v_{i}$ for each $1 \leq i \leq n$. Then $A=\operatorname{diag}\left(u_{1}, u_{2}, \ldots, u_{n}\right)+B$, where $B=\left(b_{i j}\right)$ with $b_{i i}=v_{i}(1 \leq i \leq n)$ and $b_{i j}(i \neq j)=a_{i j}$. It is clear that $\operatorname{diag}\left(u_{1}, \ldots, u_{n}\right), B \in U\left(T_{n}(R)\right)$.

(2) Put $\Theta: Q M_{2}(R) \rightarrow T_{2}(R),\left(\begin{array}{ll}a & b \\ c & d\end{array}\right) \mapsto\left(\begin{array}{cc}a+b & b \\ 0 & d-b\end{array}\right)$. Then $\Theta$ is a monomorphism of rings. Also, for any $\left(\begin{array}{ll}x & z \\ 0 & y\end{array}\right) \in T_{2}(R)$, we have

$$
\Theta\left(\left(\begin{array}{cc}
x-y & z \\
x-y-z & y+z
\end{array}\right)\right)=\left(\begin{array}{ll}
x & z \\
0 & y
\end{array}\right) .
$$

Hence $\Theta$ is an isomorphism of rings. This completes the proof by (1).

(3) The proof is similar to that of (1).

(4) Note that $R[x] /\left(x^{n}\right) \cong S_{n}(R)$, we obtain the result by (3).

Given a ring $R$ and a $(R, R)$-bimodule $M$, the trivial extension of $R$ by $M$ is the $\operatorname{ring} T(R, M)=R \bigoplus M$ with the usual addition and the following multiplication:

$$
\left(r_{1}, m_{1}\right)\left(r_{2}, m_{2}\right)=\left(r_{1} r_{2}, r_{1} m_{2}+m_{1} r_{2}\right) .
$$

Proposition 3.17. $T(R, M)$ is 2-good if and only if $R$ is 2-good.

Proof. Suppose $T(R, M)$ is 2-good. For any $x \in R$, we have $(x, 0)=(u, m)+$ $(v, n)$, where $(u, m),(v, n) \in U(T(R, M))$ with inverses $\left(u_{1}, m_{1}\right)$ and $\left(v_{1}, n_{1}\right)$. Note that $1_{T}=\left(1_{R}, 0\right)$, by $(u, m)\left(u_{1}, m_{1}\right)=\left(1_{R}, 0\right)=\left(v_{1}, n_{1}\right)(v, n)$ we obtain 
$u u_{1}=1_{R}=u_{1} u, v v_{1}=1_{R}=v_{1} v$. Hence $x=u+v$ with $u, v \in U(R)$. So $R$ is 2 -good.

Conversely, suppose $R$ is 2-good. For any $(x, m) \in T(R, M)$, by hypothesis, there exist $u, v \in U(R)$ such that $x=u+v$. Thus $(x, m)=(u, m)+(v, 0)$. Since $(u, m)\left(u^{-1},-u^{-1} m u\right)=(1,0)$ and $(v, 0)\left(v^{-1}, 0\right)=(1,0),(u, m),(v, 0) \in$ $U(T(R, M))$. Hence $T(R, M)$ is 2 -good.

Let $R$ be a commutative ring, $M$ be an $R$-module, and $\sigma$ be an endomorphism of $R$. Give $R \bigoplus M$ a ring structure with multiplication $\left(r_{1}, m_{1}\right)\left(r_{2}, m_{2}\right)$ $=\left(r_{1} r_{2}, \sigma\left(r_{1}\right) m_{2}+r_{2} m_{1}\right)$, where $r_{i} \in R$ and $m_{i} \in M$. We call this extension the Nagata extension of $R$ by $M$ and $\sigma$ and denote it by $N(R, M, \sigma)$.

Proposition 3.18. $N(R, M, \sigma)$ is 2-good if and only if $R$ is 2-good.

Proof. Suppose $R$ is 2-good. Then for any $(x, m) \in N(R, M, \sigma)$ there exist $u, v \in U(R)$ such that $(x, m)=(u, m)+(v, 0)$. Since

$$
(u, m)\left(u^{-1},-\sigma\left(u^{-1}\right) m u^{-1}\right)=\left(1_{R}, 0\right)
$$

and $(v, 0)\left(v^{-1}, 0\right)=(1,0),(u, m),(v, 0) \in U(N(R, M, \sigma))$. Hence $N(R, M, \sigma)$ is 2 -good.

The converse is similar to Proposition 3.17.

A ring $R$ is called right ore if given $a, b \in R$ with $b$ regular there exist $a_{1}, b_{1} \in R$ with $b_{1}$ regular such that $a b_{1}=b a_{1}$. It is a well-known fact that $R$ is a right ore ring if and only if the classical right quotient ring of $R$ exists.

Proposition 3.19. Let $R$ be a right ore ring and $Q$ be the classical right quotient ring of $R$. If $R$ is 2-good, then so is $Q$.

Proof. For any $r=a b^{-1} \in Q$, where $a, b \in R$ with $b$ regular. By hypothesis there exist $u, v \in U(R)$ such that $a=u+v$. Hence $r=u b^{-1}+v b^{-1}$. It is clear that $\left(u b^{-1}\right)^{-1}=b u^{-1},\left(v b^{-1}\right)^{-1}=b v^{-1}$, thus $u b^{-1}, v b^{-1} \in U(Q)$.

The converse of Proposition 3.19 is not true. For example, the rational number field $Q$ is the classical right quotient ring of $\mathbb{Z}$, but $\mathbb{Z}$ is not 2 -good.

Let $R$ be an algebra over a commutative ring $S$. Recall that the Dorroh extension of $R$ by $S$ denoted $D(R, S)$, is the ring $R \times S$ with operations $\left(r_{1}, s_{1}\right)+$ $\left(r_{2}, s_{2}\right)=\left(r_{1}+r_{2}, s_{1}+s_{2}\right)$ and $\left(r_{1}, s_{1}\right)\left(r_{2}, s_{2}\right)=\left(r_{1} r_{2}+s_{1} r_{2}+s_{2} r_{1}, s_{1} s_{2}\right)$, where $r_{i} \in R$ and $s_{i} \in S$.

Proposition 3.20. The Dorroh extension $D(R, S)$ of $R$ by $S$ is 2-good if the following conditions are satisfied:

(1) $S$ is 2-good;

(2) $R$ is right quasi-regular.

Proof. Assume that (1), (2) are satisfied. Let $d=(r, s) \in D(R, S)$. Then by (1), we can write $s=u+v$ with $u, v \in U(S)$. Thus $d=(r, u)+(0, v)$ and $(0, v)$ is unit since $(0, v)\left(0, v^{-1}\right)=(0,1)$. Now we have $(r, u)=(0, u)\left(u^{-1} r, 1\right)$, and 
$\left(u^{-1} r, 1\right)=(0,1)+\left(u^{-1} r, 0\right)$ is a unit of $D(R, S)$ because $(R, 0) \subseteq J(D(R, S))$ by (2). Hence $(r, u) \in U(D(R, S))$, so $d$ is 2-good, as required.

\section{References}

[1] A. Badawi, On abelian $\pi$-regular rings, Comm. Algebra 25 (1997), no. 4, 1009-1021.

[2] _ On semicommutative $\pi$-regular rings, Comm. Algebra 22 (1994), no. 1, 151157.

[3] V. Camillo and P. P. Nielsen, McCoy rings and zero-divisors, J. Pure Appl. Algebra 212 (2008), no. 3, 599-615.

[4] V. P. Camillo and H. P. Yu, Exchange rings, Units and idempotents, Comm. Algebra 22 (1994), no. 12, 4737-4749.

[5] H. Chen, Units, idempotents, and stable range conditions, Comm. Algebra 29 (2001), no. 2, 703-717.

[6] P. Crawley and B. Jonsson, Refinements for infinite direct decompositions of algebraic systems, Pacific J. Math. 14 (1964), 797-855.

[7] G. Ehrlich, Unit-regular rings, Port. Math. 27 (1968), 209-212.

[8] J. W. Fisher and R. L. Snider, Rings generated by their units, J. Algebra 42 (1976), no. $2,363-368$.

[9] L. Gillman and M. Jerison, Rings of Continuous Functions, The University Series in Higher Mathematics, D. Van Nostrand Co., Princeton, N. J., 1960.

[10] K. R. Goodearl and P. Menal, Stable range one for ring with many units, J. Pure Appl. Algebra 54 (1988), no. 2-3, 261-287.

[11] M. Henriksen, Two classes of rings generated by their units, J. Algebra 31 (1974), $182-193$

[12] A. V. Kelarev, Ring Constructions and Applications, World Scientific, New Jersey, 2002.

[13] W. K. Nicholson, Lifting idempotents and exchange rings, Trans. Amer. Math. Soc. 229 (1977), 269-278

[14] J. Okninski, Semigroup Algebras, Marcel Dekker, INC, New York, 1991.

[15] R. Raphael, Rings which are generated by their units, J. Algebra 28 (1974), 199-205.

[16] J. Stock, On rings whose projective modules have the exchange property, J. Algebra 103 (1986), no. 2, 437-453.

[17] A. A. Tuganbaev, Rings and modules with exchange properties, J. Math. Sci. (N.Y.) 110 (2002), no. 1, 2348-2421.

[18] P. Vámos, 2-good rings, Q. J. Math. 56 (2005), no. 3, 417-430.

[19] L. N. Vaserstein, Bass's first stable range condition, J. Pure Appl. Algebra 34 (1984), no. 2-3, 319-330.

[20] R. B. Warfield, Exchange rings and decomposition of modules, Math. Ann. 199 (1972), 31-36.

[21] G. S. Xiao and W. T. Tong, n-clean rings, Algebra Colloq. 13 (2006), no. 4, 599-606.

[22] Y. Q. Ye, Semiclean rings, Comm. Algebra 31 (2003), no. 11, 5609-5625.

[23] H. P. Yu, On the structure of exchange rings, Comm. Algebra 25 (1997), no. 2, 661-670.

[24] B. Zimmermann-Huisgen and W. Zimmermann, Classes of modules with the exchange property, J. Algebra 88 (1984), no. 2, 416-434.

YAO WANG

SChool of Mathematics and Statistics

NANJing University of Information Science and Technology

NANJing 210044, P. R. ChinA

E-mail address: wangyao@nuist.edu.cn 
YANLI REN

School of Mathematics and Information Technology

NANJing XiaOZhuang University

NAnjing 211171, P. R. China

E-mail address: renyanlisx@163.com 